\title{
Modeling a Battery-Electric Three-Wheeled Car Concept
}

\author{
$\underline{\text { Rebecca Margetts }}$ \\ University of Lincoln, School of Engineering, Brayford Pool, Lincoln LN6 7TS. UK \\ Donald Margolis
}

University of California Davis, Dept. Mechanical and Aerospace Engineering, 1 Shields Ave, Davis, CA 95616, USA

Copyright $\odot$ 20xx SAE International

\begin{abstract}
This paper describes a multi-degree-of-freedom model of a threewheeled car, implemented in Matlab®. The purpose was to investigate the dynamics of the car (assumed to be rigid on its suspension) during cornering. While the problems associated with three-wheeled cars are well-known, much of the guidance in the literature and off-the-software assumes a conventional four-wheeled car. Consequently, the authors were approached with a batteryelectric concept car which was thought to offer better performance than existing variants, because the use of hub motors lowered the centre of gravity and hence reduced rollover coefficient. However, simulation of the vehicle model in cornering shows that the concept is still prone to instability. Indeed, it suffers greater roll velocities than a comparable three-wheeled car with internal combustion engine, because the ratio of sprung to unsprung mass is significantly altered. This paper therefore recommends a programme of further simulations and model-based design changes to progress the concept to a marketable performance product.
\end{abstract}

\section{Introduction}

Three-wheeled cars have been present throughout the history of the automobile. Indeed, some of the earliest cars had three wheels, such as the Benz Patent Motorwagen. Reducing the number of wheels offers a reduction in weight and - depending on the configuration - a simplified steering mechanism, yielding an extremely lightweight and affordable vehicle. As the automotive industry moves towards a new generation of light, efficient, and electrified vehicles, it is timely to revisit the three-wheeled car.

This paper relates specifically to a concept vehicle: the Mibrid Mayfly. This is a lightweight battery-electric car designed for the roads of rural Britain. The British commuter typically lives outside the city, necessitating a car with a higher top speed and battery range than conventional metropolitan electric vehicles, but still needs to handle well on roads that are smaller than a conventional highway. The concept is one of a 'Gentleman Racer,' evoking classic British cars such as the MG Midget which perform well but are accessible to the average road user. The term 'Gentleman Racer' is a little outdated, so we use the more inclusive term 'Aspirational Racecar.' The design utilises a Reliant Robin chassis: a car which again has a cult following in the UK and evokes 'quirky' British design. By using the Reliant Robin as a basis, the development team committed to a 'delta' configuration (one wheel at the front and two at the rear). Modern three-wheeled vehicles (such as those produced by Morgan and Elio) tend to favour a 'tadpole' configuration (two wheels at the front and one at the rear). 'Tadpole' vehicles offer better stability and aerodynamics than 'Delta' vehicles, but require a more complicated (and hence heavier) steering system and can be less aesthetically appealing. Some Delta vehicles are still under development or production, but they largely incorporate a tilting front wheel (such as the Trivelo E-lisa) or 'tilting' system (like CLEVER [1]), allowing the single front wheel to behave like that of a motorbike and lean during cornering.

The Mayfly concept had postulated that the lower center of gravity (produced by using hub motors and a floorpan-mounted battery) would give the Mayfly superior performance to existing designs such as the reliant Robin. A 2018 pilot study [2] was conducted into the dynamics of this vehicle, in order to establish if this was indeed the case and establish the extent to which performance is improved. A Reliant Robin of similar size and mass was used as a benchmark. The pilot study did indeed indicate that quasi-static rollover and understeer coefficient were improved compared to the Reliant Robin. However, it also revealed that much of the existing guidance [3] and off-theshelf modelling and simulation software [4] was intended for fourwheeled cars. As such, they incorporate assumptions which did not allow for the complex and unique dynamics of a three-wheeled car, and indicated a stable performance which contradicts engineering experience with this type of vehicle. It is commonly accepted that delta-style three-wheeled cars (i.e. those with one wheel at the front) can easily go unstable, and a rolling/tipping motion and 'spinning out' can be observed when cornering at even fairly low speeds.

Hence, this further study builds a three-wheeled car model from first principles in order to fully capture the dynamics of the vehicle in cornering. Selected design parameters were optimised, in order to establish the best achievable performance from this concept. This will then be assessed and used to suggest further design changes to the concept.

\section{Goals and Objectives}

This paper describes a three-wheeled car model. The lower-frequency dynamics are captured by considering the rigid body on the suspension, with vertical, lateral and fore/aft dynamics of the tires. This was chosen over the usual 'bicycle model' assumption used for steady state cornering analyses, because some pitching motion can be observed in delta vehicles when they roll over. The model was parameterised so as to compare the Mayfly concept with a similar ICE delta car: the Reliant Robin. The model yields results consistent with 
experience, and uses that model to recommend improvements to the Mayfly concept.

\section{Methodology}

\section{Overview}

A model was generated from the system itself using bond graphs [5]. This method allows the practitioner to construct a model from first principles, using the physics of a system as a guide. It then facilitates the generation of a mathematical model in state space format, which can be transferred to a computer software package. In this case, Matlab® was selected due to its accessibility.

The model makes different assumptions to those of a regular fourwheeled car. Rather than assuming a 'bicycle model' in steady-state cornering, this model incorporates all three wheels and captures pitch movement. This is because three-wheeled cars can be seen to have a roll/pitch 'tipping' motion in cornering, rather than a pure rolling motion. In addition, a Dugoff tire model is used instead of the usual linear tire assumption, because the single front wheel quickly exceeds the limit of proportionality in cornering.

A limited amount of data exists for the Mibrid Mayfly since it is still in the concept stage: the Mayfly has a target mass of $<500 \mathrm{~kg}$ and will use off-the-shelf brushless DC in-wheel hub motors [6]. However, the concept is based on a Reliant Robin chassis, so dimensional data for the 1981 Robin 850 - which is readily available [7] - was used. The wheels and suspension are dated, with 12" wheels and a soft leaf suspension. Consequently, the authors took the liberty of assuming 14 " wheels and a ride frequency consistent with modern cars.

The Robin 850 was also used as a benchmark in analyses, to show how the Mayfly performs compares to a once-popular three-wheeled car which is - for many people - synonymous with delta style cars.

\section{Model}

The model consists of a multi-degree of freedom rigid vehicle body, attached to three suspension models. These suspension models comprise a classic quarter-car model (or, in this case, a third-car) to capture the vertical dynamics, a lateral tire model to capture lateral dynamics, and a longitudinal tire model to capture fore/aft dynamics. These submodels are reused, but contain different parameters depending on whether they are front or rear due to the differences in front and rear suspension. Linear suspension components are assumed, and a Dugoff tire model is used [8]. Figure 1 shows the high level model, and Figure 2 - Figure 3 detail the submodels as schematics. Figure 4 illustrates some dimensions and directions used in the model.

The equations obtained for the system are as follows. Velocities of each wheel (front $f$, rear right $r r$ and rear left $r l$ ):

$$
\begin{aligned}
& U_{f}=U-\left(h_{g}-R_{w}\right) \omega_{p} \\
& V_{f}=V+\left(h_{g}-R_{w}\right) \omega_{r}+a \omega_{y}-U \delta \\
& W_{f}=W-a \omega_{p}
\end{aligned}
$$

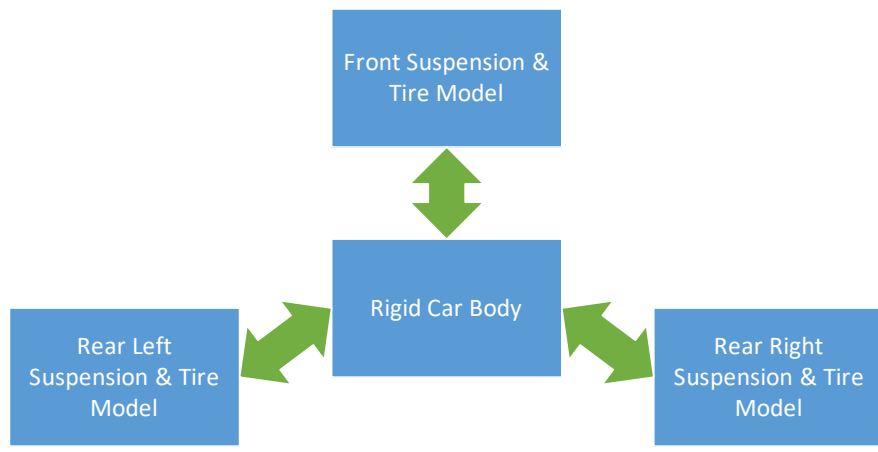

Figure 1: High Level Model

$$
\begin{aligned}
& U_{r r}=U-\left(h_{g}-R_{w}\right) \omega_{p}+\frac{w}{2} \omega_{y} \\
& V_{r r}=V+\left(h_{g}-R_{w}\right) \omega_{r}-b \omega_{y} \\
& W_{r r}=W-\frac{w}{2} \omega_{r}+b \omega_{p} \\
& U_{l r}=U-\left(h_{g}-R_{w}\right) \omega_{p}-\frac{w}{2} \omega_{y} \\
& V_{l r}=V+\left(h_{g}-R_{w}\right) \omega_{r}-b \omega_{y} \\
& W_{l r}=W+\frac{w}{2} \omega_{r}+b \omega_{p}
\end{aligned}
$$

Velocities are calculated by finding the incremental difference in displacements $q$ with each time step. Those associated with the suspension (i.e. vertical) on wheel unit $n$ are:

$$
\begin{aligned}
& d q_{s_{n}}=v_{u s_{n}}-W_{n} \\
& d q_{t_{n}}=v_{g_{n}}-v_{u s_{n}}
\end{aligned}
$$

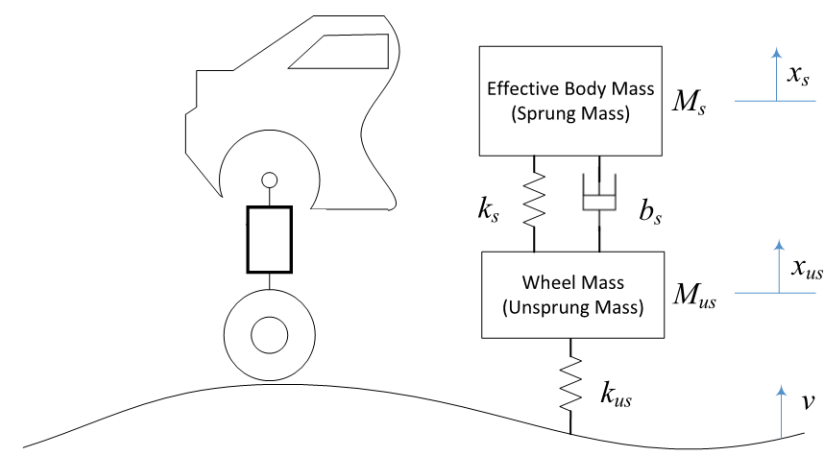

Figure 2: The 'Quarter Car' Model i.e. Vertical Dynamics 


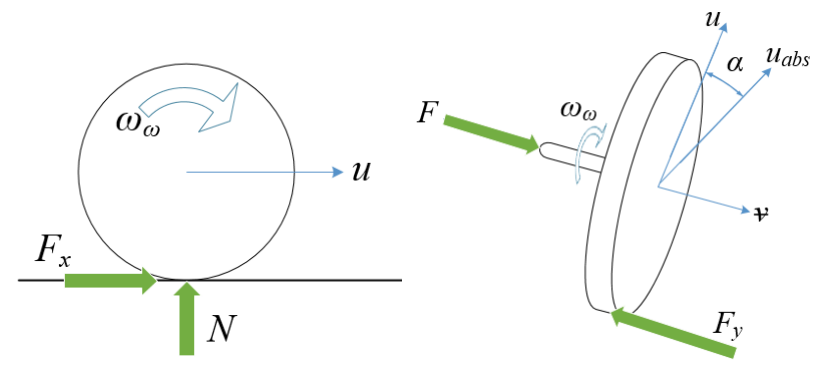

Figure 3: Longitudinal [left] and Lateral [right] Tire Dynamics

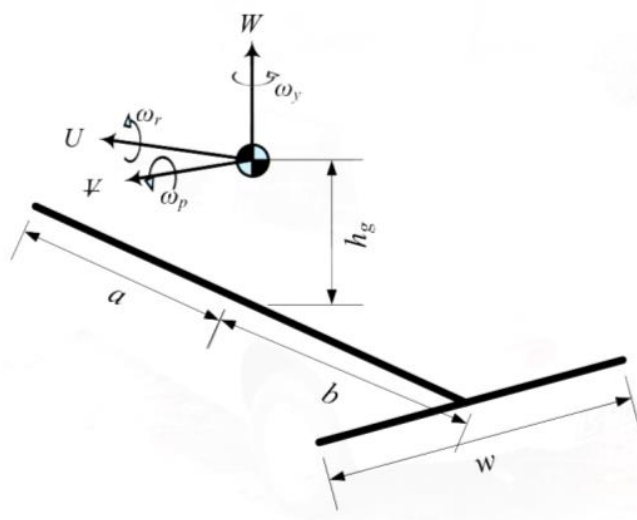

Figure 4: Dimensions and directions on the Delta car chassis

Tire lateral slip model:

$\alpha_{n}=-\frac{V_{n}}{U_{n}}$

For the front tire:

$s_{f}=0$

And for the rear tires:

$S_{n}=\frac{R_{w} \omega_{w n}-U_{n}}{\left|U_{n}\right|}$

Then, for each tire $n$ :

$$
\begin{aligned}
& \mu_{n}=\mu(0)\left(1-\varepsilon U\left|s_{n}\right|\right) \\
& \lambda_{n}=\frac{\mu_{n} N_{n}\left(1-\left|s_{n}\right|\right)}{2 / \sqrt{\left(C_{x_{n}} s_{n}\right)^{2}+\left(C_{y_{n}} \tan \alpha_{n}\right)^{2}}}
\end{aligned}
$$

Yielding forces:

$$
\begin{aligned}
& F_{x d_{n}}=C_{x_{n}} s_{n} /\left(1-\left|s_{n}\right|\right) \\
& F_{y d_{n}}=C_{y_{n}} \tan \alpha_{n} /\left(1-\left|s_{n}\right|\right)
\end{aligned}
$$

$$
F_{x_{n}}=\mid \begin{array}{cc}
F_{x d_{n}} & \lambda_{n}>1 \\
F_{x d_{n}} 2 \lambda_{n}\left(1-\frac{\lambda_{n}}{2}\right) & \lambda_{n} \leq 1 \\
F_{y d_{n}} & \lambda_{n}>1 \\
F_{y_{n}}=\mid \begin{array}{cc}
2 \lambda_{n}\left(1-\frac{\lambda_{n}}{2}\right) & \lambda_{n} \leq 1
\end{array}
\end{array}
$$

The vertical forces (associated with the suspension) are:

$$
F_{s_{n}}=k_{s_{n}} q_{s_{n}}+b_{s_{n}} d q_{s_{n}}
$$

Changes in force $p$ with each time step are:

$$
\begin{aligned}
d p_{V}= & -m_{s} \omega_{y} U+m_{s} \omega_{r} W+\sum F_{y_{n}} \\
d p_{W}= & m_{s} \omega_{p} U-m_{s} \omega_{r} V+\sum F_{s_{n}}-m_{s} g \\
d p_{J_{r}} & =\left(J_{p}-J_{y}\right) \omega_{p} \omega_{y}+\left(h_{g}-R_{w}\right) \sum F_{y_{n}} \\
& +\left(F_{s_{l r}}-F_{s_{r r}}\right) \frac{w}{2} \\
d p_{J_{p}} & =\left(J_{y}-J_{r}\right) \omega_{y} \omega_{r}-F_{s_{f}} a \\
& -\left(F_{x_{r r}}+F_{x_{l r}}\right)\left(h_{g}-R_{w}\right)+\left(F_{s_{r r}}+F_{s_{l r}}\right) b-\tau_{r r}-\tau_{l r} \\
d p_{J y} & =\left(J_{r}-J_{p}\right) \omega_{r} \omega_{p}+F_{y_{f}} a-\left(F_{y_{r r}}+F_{y_{l r}}\right) b \\
& +\left(F_{x_{r r}}+F_{x_{x_{r}}}\right) \frac{w}{2} \\
d p_{u s_{n}} & =N_{n}-F_{s_{n}}-m_{u s_{n}} g
\end{aligned}
$$

Then, for the rear wheels:

$$
d p_{J \omega_{n}}=\tau_{n}-F_{x_{n}} R_{w}
$$

At the center of mass: 


$$
\begin{aligned}
& d X=U \cos \theta_{y}-V \sin \theta_{y} \\
& d Y=U \sin \theta_{y}+V \cos \theta_{y} \\
& d \theta_{y}=\omega_{y} \\
& d \theta_{r}=\omega_{r} \\
& d \theta_{p}=\omega_{p}
\end{aligned}
$$

The system of equations is then integrated by Euler over a time period.

\section{Caveats and Assumptions}

Conventional front-wheel steering is assumed, which may not be the case with the final Mayfly: the use of hub motors on all wheels means that an element of differential steering could be incorporated to improve performance. These analyses are therefore a little conservative.

The vehicle body is assumed to be rigid, which will hold true at the low frequencies under investigation here. This distinction may not be as clear-cut with the Mayfly, which will almost certainly have a stiff suspension (typically yielding a ride frequency of $\sim 15 \mathrm{~Hz}$ ) to give it racecar-like handling, and an extremely lightweight composite body which could prove more flexible than the norm. Further modelling is therefore recommended during the design phase of the chassis and body.

\section{Results}

The model was used to simulate the vehicle in a steady-state corner. The forward speed of the vehicle $U$ and the steering angle of the front wheel $\delta$ were varied to explore how they affect the dynamic behaviour of the vehicle.

\section{Establishing a Maximum Velocity \& Steering Angle}

The Mayfly concept is one of an aspirational racer, which a customer may well use at a track day as well as being road legal. Consequently, initial simulation was conducted up to speeds of $50 \mathrm{mph}$ (bearing in mind that a Formula 1 car corners at around 70mph) and at a steering angle of 12 degrees (with 'full lock' being around 25 degrees).

Figure 5 and Figure 6 show how vehicle position and lateral acceleration vary depending on the steering angle, at a vehicle velocity of 50mph. At lower steering angles (up to $0.1 \mathrm{rad}$, or 5.7 degrees) the car follows an expected curve, and experiences an initial peak lateral acceleration which quickly converges to a constant value. At higher steering angles, the vehicle positioning does not change with steering angle but the lateral acceleration is oscillating: this indicates that the vehicle is close to instability, and is no longer responding to the steering input.

A similar study, during which the steering angle was kept constant at 12 degrees and the velocity varied, is shown in Figure 7. In Figure 7 there is a distinct point at which the vehicle appears to stop adhering to the expected curve and skids, and this point occurs at $13.5 \mathrm{~m} / \mathrm{s}$ (30mph). Beyond this speed, the path of the vehicle in the corner straightens.

\section{Performance at Reasonable Velocity \& Steering Angle}

Simulation at a speed of $35 \mathrm{mph}$ and steering angle of 6 degrees gives more results in the region of interest (i.e. prior to skidding) and shows how the vehicle can be expected to perform in a more usual 'maximum' condition. The vehicle follows a curve as shown in Figure 8 , consistent with expectation for a steady-state corner. The velocities in Figure 9 - Figure 11 quickly reach a stable steady-state, as do the roll, pitch, and slip angles (Figure 12 - Figure 14). Normal and lateral forces on the tires (Figure 15 - Figure 16) indicate the vehicle is leaning towards left, consistent with the roll angle. The lateral slip angle and lateral force on the front tire is distinctly higher than those of the rear tires - which is to be expected. Lateral acceleration (Figure 17) oscillates very slightly, but is stable with a peak value of $0.6 \mathrm{~g}$. This is well within the operational limits of the tire model.

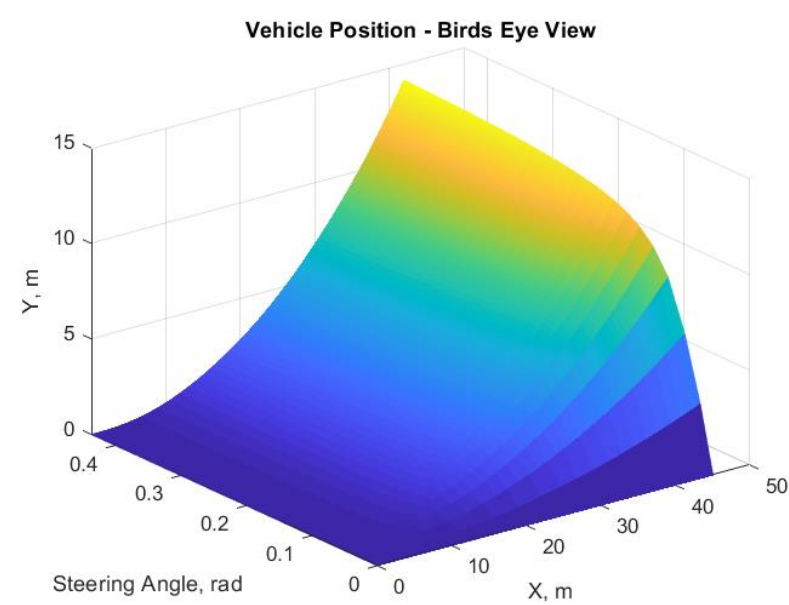

Figure 5: Vehicle position during a corner at 50mph, with varying steering angle

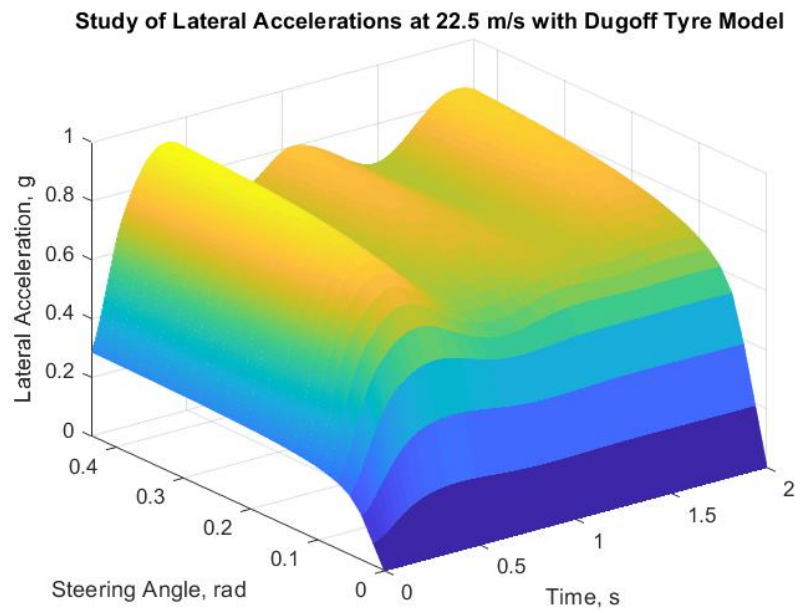

Figure 6: Lateral acceleration during a corner at $50 \mathrm{mph}$, with varying steering angle 


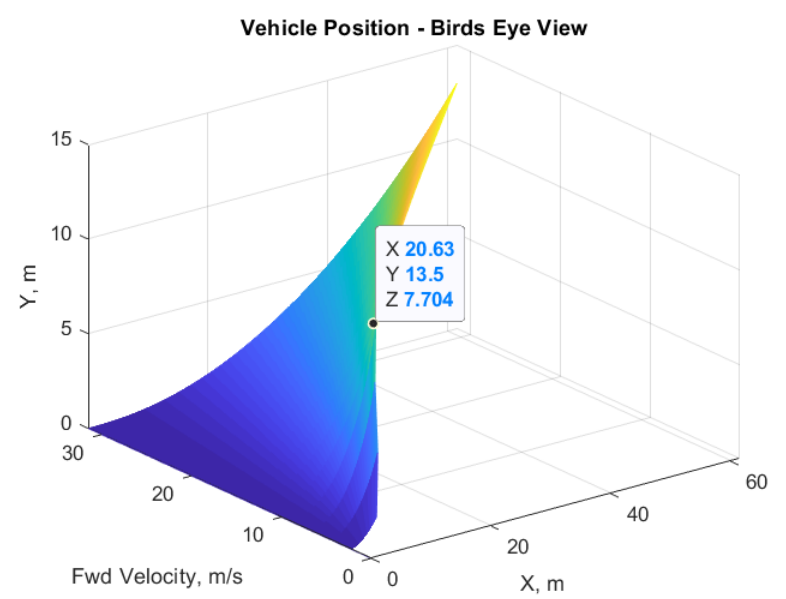

Figure 7: Vehicle position during a 12 degree corner, with varying velocity

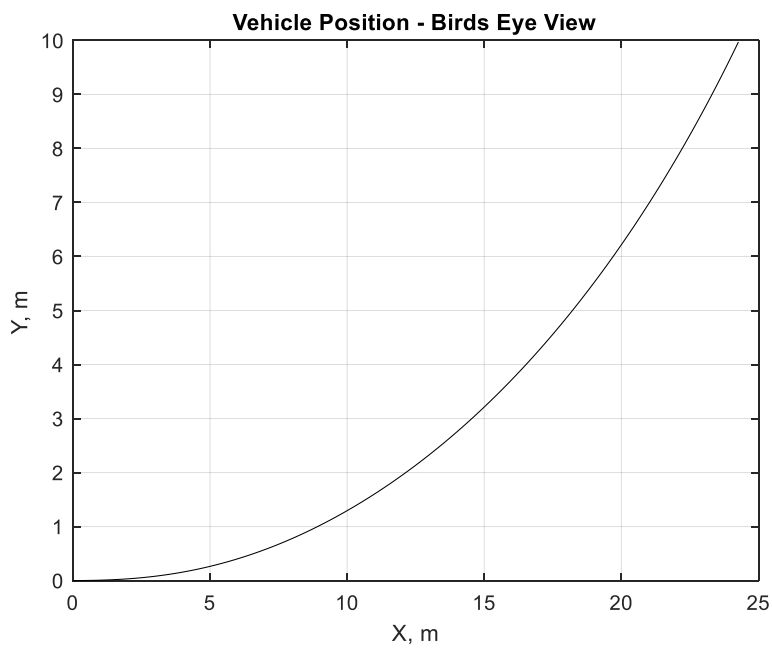

Figure 8: Vehicle Position in 6deg corner at 30mph

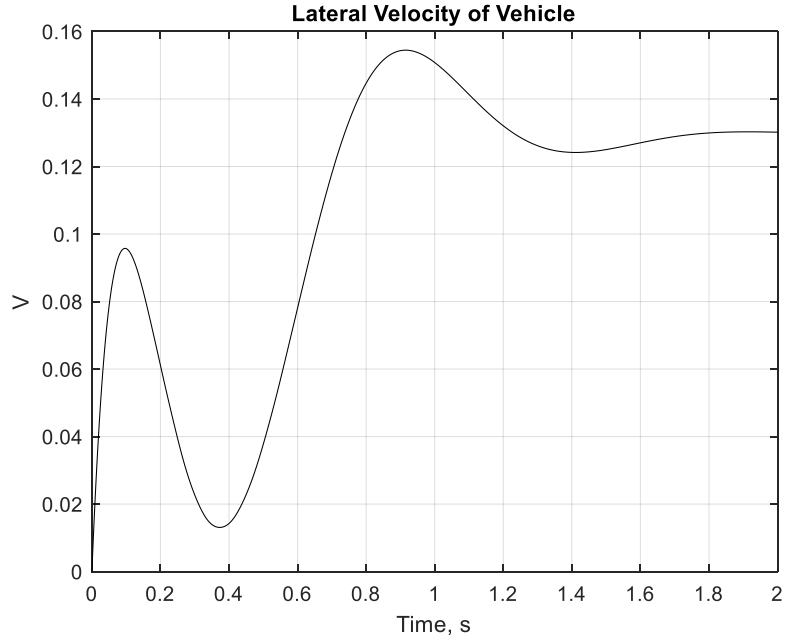

Figure 9: Lateral Velocity in 6deg corner at 30mph

Page 5 of 9

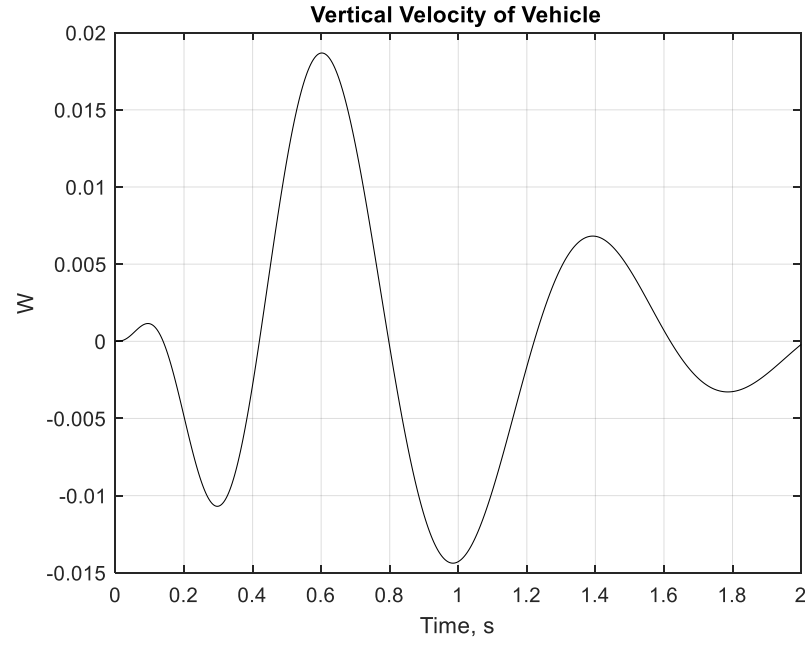

Figure 10: Vertical Velocity in 6deg corner at 30mph

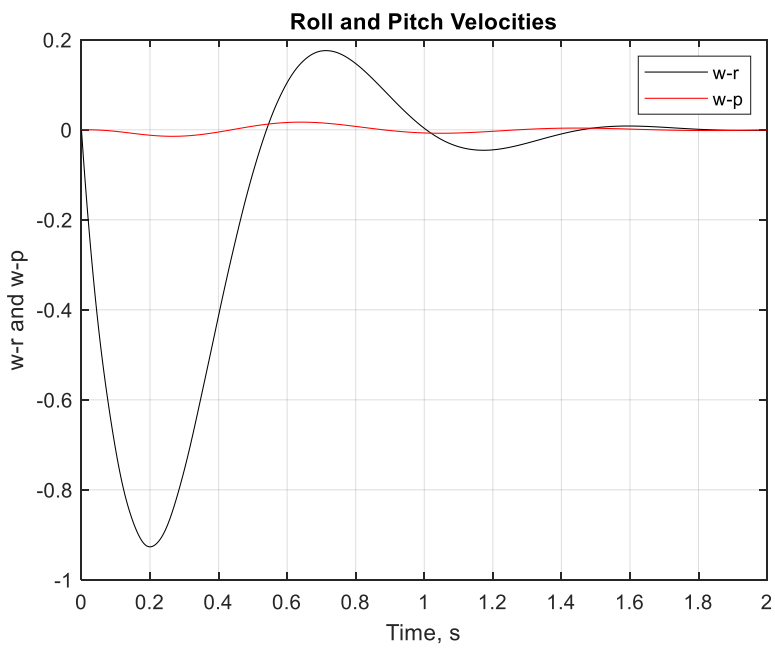

Figure 11: Roll and Pitch Velocities in 6deg corner at 30mph

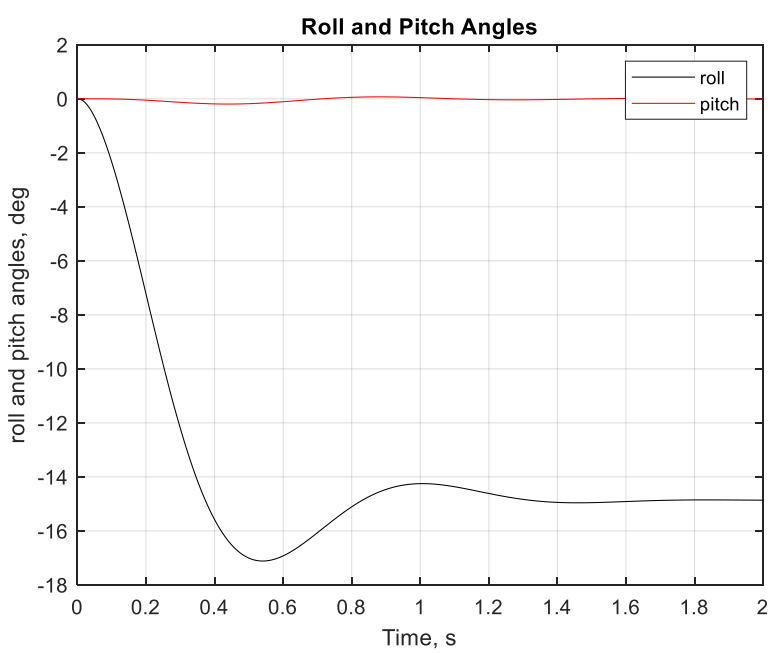

Figure 12: Vehicle Roll \& Pitch Angles in 6deg corner at 30mph 


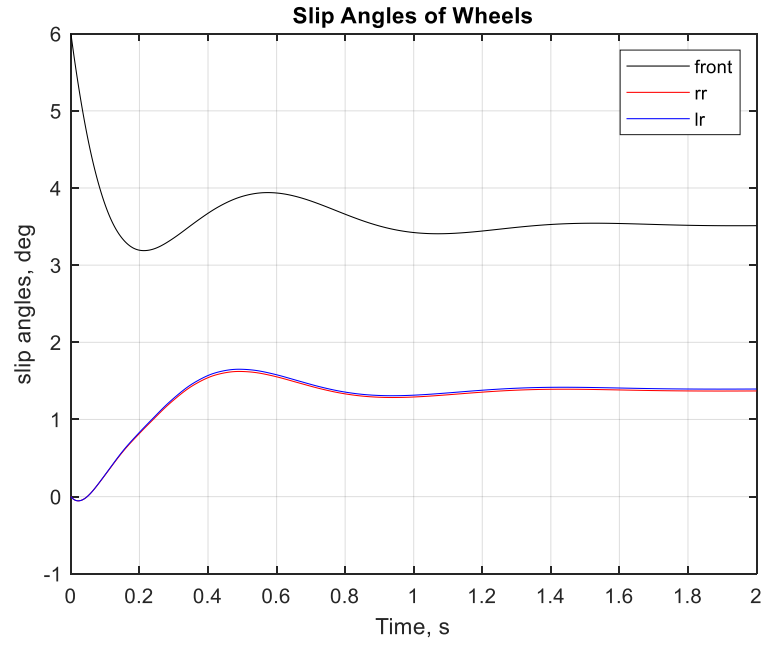

Figure 13: Lateral Slip Angles in 6deg corner at 30mph

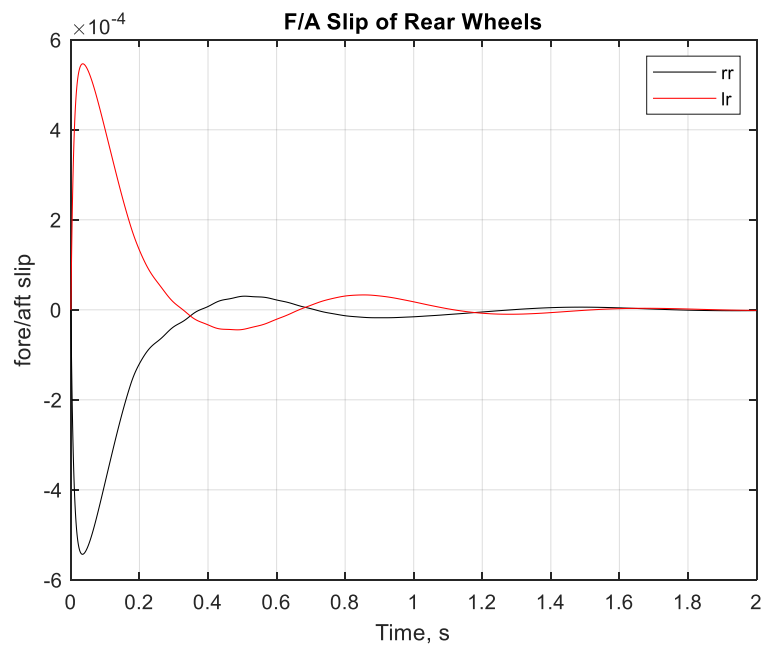

Figure 14: Fore/Aft Slip Angles in 6deg corner at 30mph

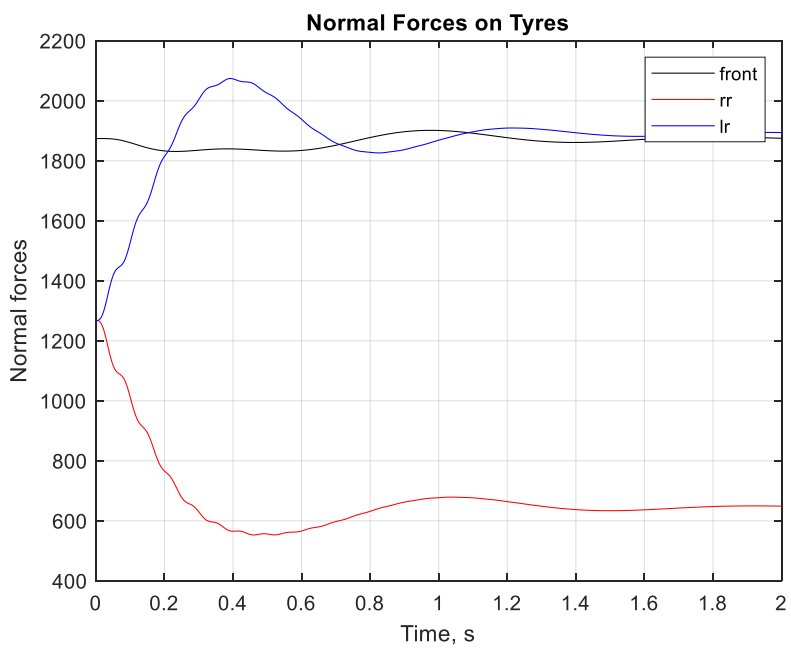

Figure 15: Normal Forces on Tires in 6deg corner at 30mph

Page 6 of 9

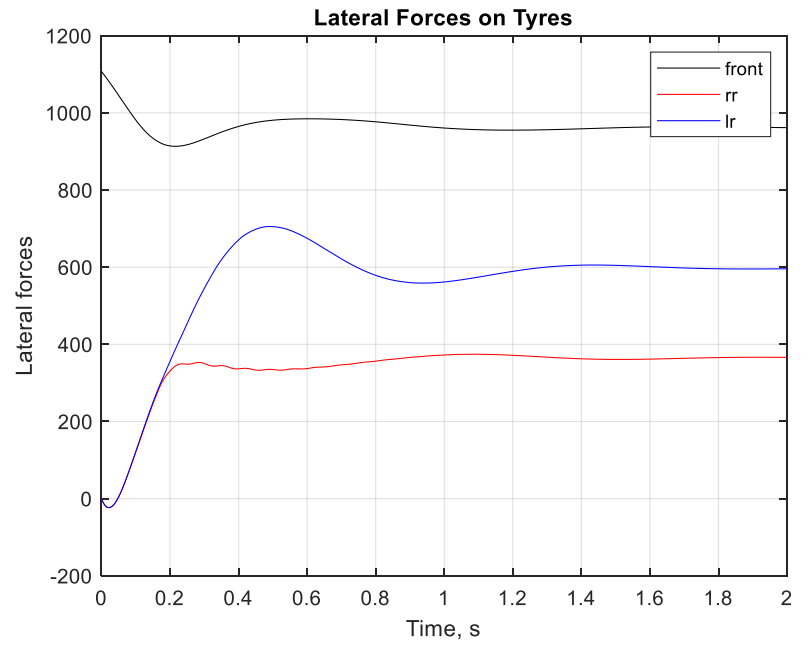

Figure 16: Lateral Forces on Tires in 6deg corner at 30mph

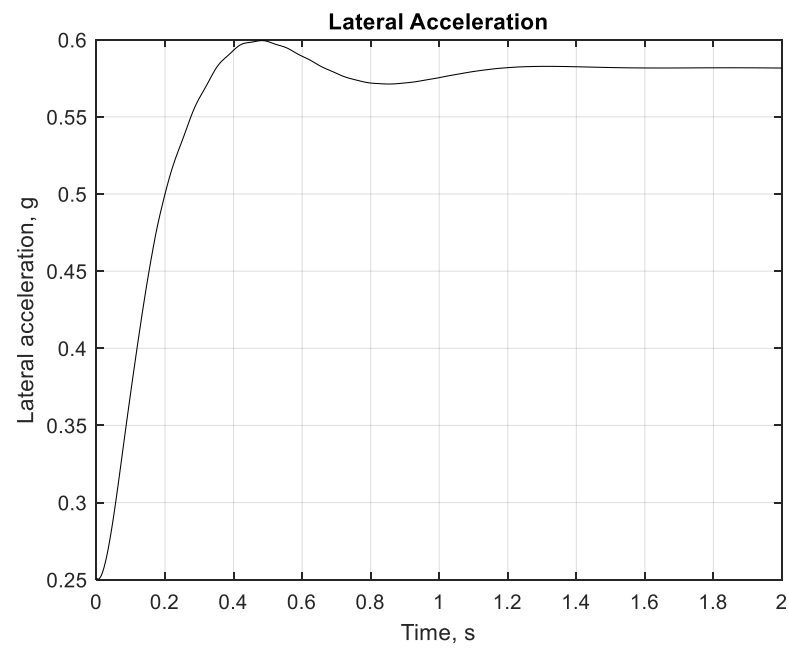

Figure 17: Vehicle Lateral Acceleration in 6deg corner at 30mph

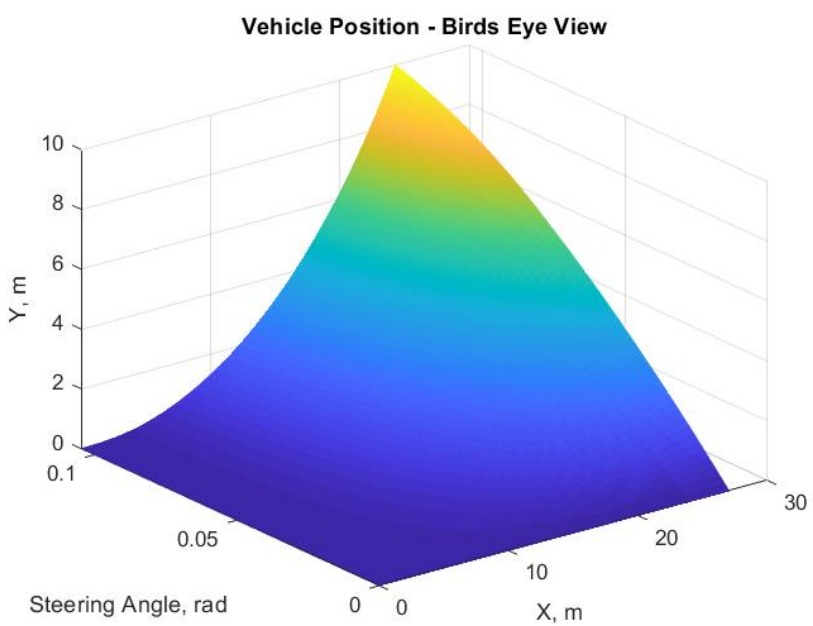

Figure 18: Vehicle position during a corner at 30mph, with varying steering angle 


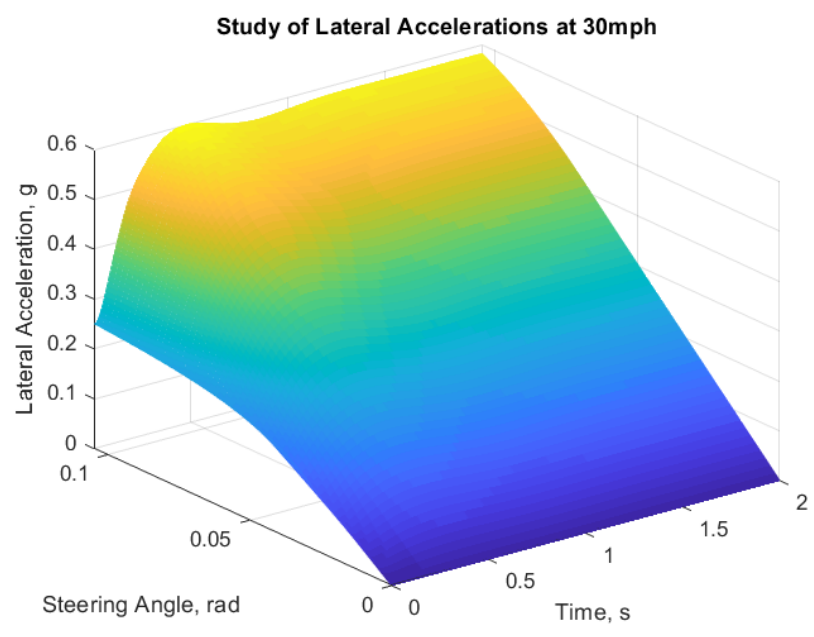

Figure 19: Lateral acceleration during a corner at $50 \mathrm{mph}$, with varying steering angle

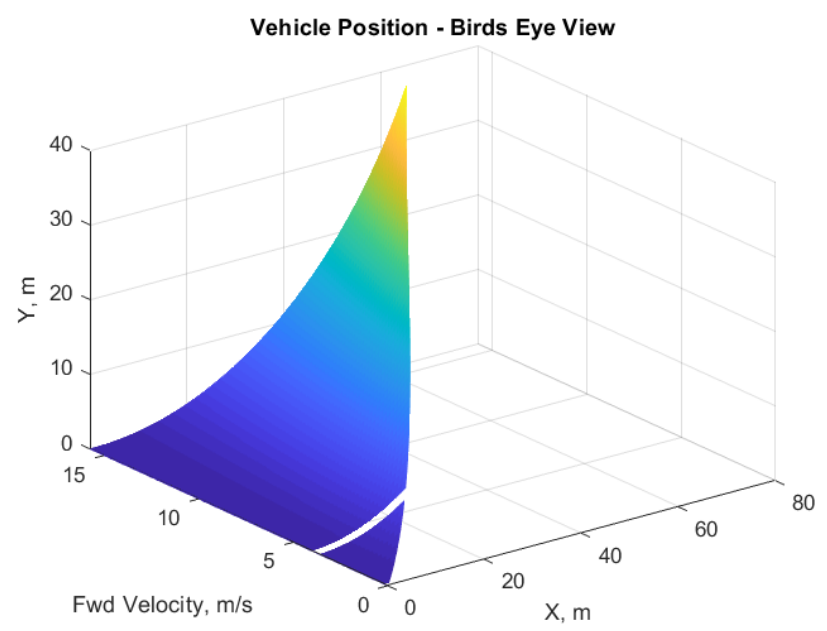

Figure 20: Vehicle position during a 3 degree corner, with varying velocity

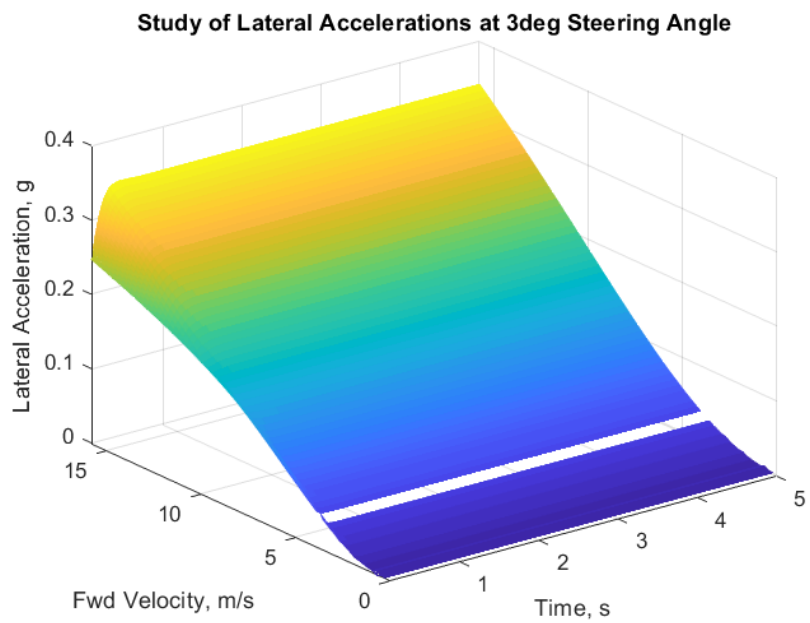

Figure 21: Lateral acceleration during a 3 degree corner, with varying velocity.

\section{Comparison with Reliant Robin}

Figure 22 - Figure 24 plot salient data for a Reliant Robin 850 for comparison with that of the Mayfly concept. Recall that the Robin has an identical chassis but significantly different weight distribution, including a lower sprung:unsprung mass ratio. During a 6 degree steady-sate corner at $30 \mathrm{mph}$ the vehicle follows the same trajectory as the Mayfly. However, it experiences a peak roll velocity in excess of $0.5 \mathrm{rad} / \mathrm{s}$, with a peak roll angle of nearly 9 degrees and reaching a steady state roll angle of around 7.5 degrees. The Mayfly model rolls in the other direction, experiencing a peak roll velocity of $0.9 \mathrm{rad} / \mathrm{s}$, with a peak roll angle of 17 degrees and reaching a steady state roll angle of around 15 degrees. Rather than yielding better performance, the Mayfly actually rolls more severely than the Robin it was intended to replace.

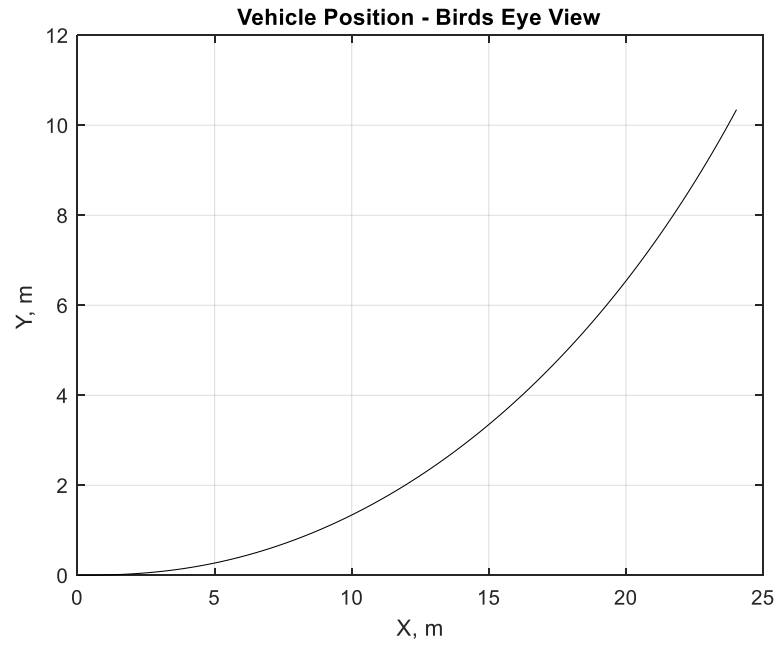

Figure 22: Vehicle Position in 6deg corner at 30mph (Reliant Robin)

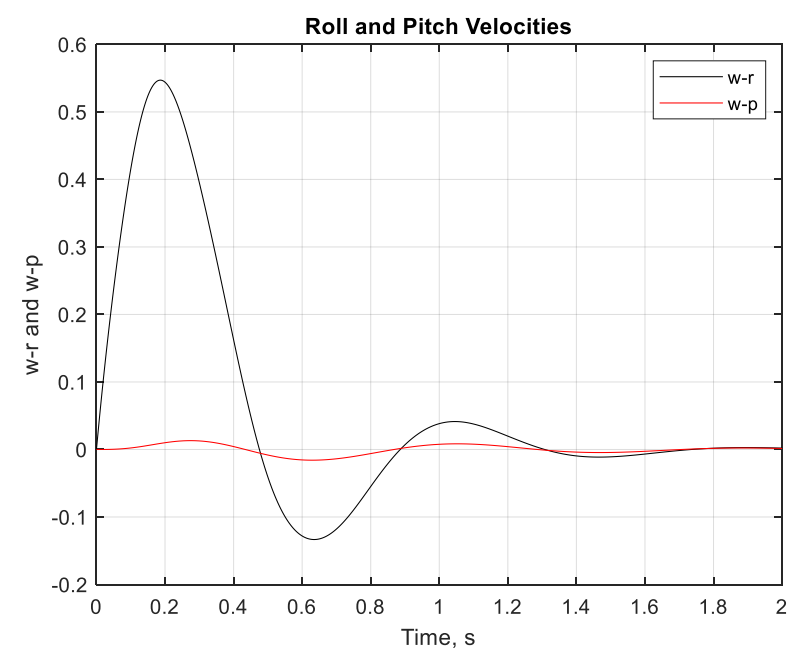

Figure 23: Vehicle Roll \& Pitch Velocity in 6deg corner at 30mph (Reliant Robin) 


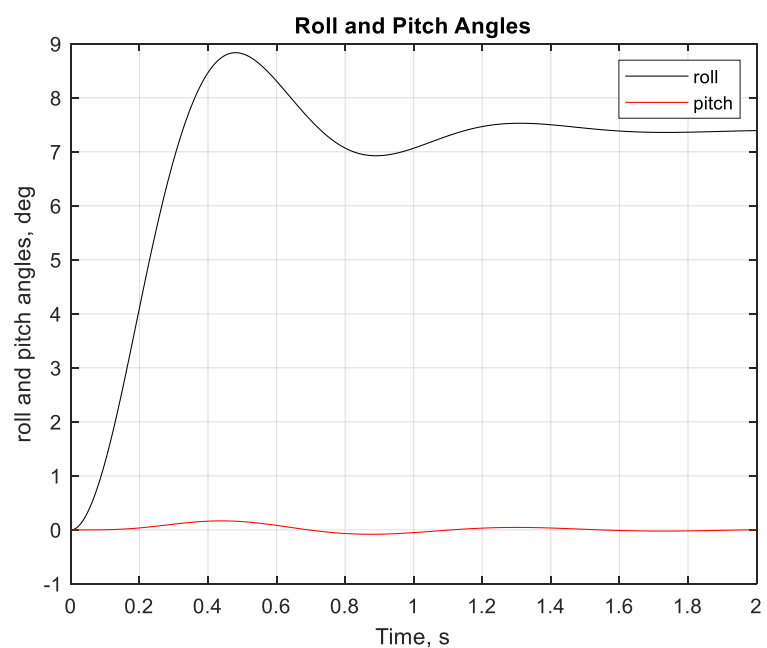

Figure 24: Vehicle Roll \& Pitch Angles in 6deg corner at 30mph (Reliant Robin)

\section{Discussion}

The three-wheeled vehicle rolls during cornering, which is consistent with experience. However, the combined roll/pitch 'tipping' motion hypothesised is not evident in the results: the pitch angles and velocities are negligible compared to the roll.

The vehicle handles corners of up to 6 degrees / $30 \mathrm{mph}$. At higher speeds and steering angles, the vehicle appears to skid: forces and slip angles on the tires saturate, and the trajectory of the car straightens. This could be a problem if the Mayfly is marketed as an aspirational racecar: it does not corner like a racecar would, and any attempt to corner at high speeds and/or tight angles could prove dangerous.

A comparison of the Mayfly model with a model of the Reliant Robin 850 (which shares a chassis) shows that the Mayfly is subject to more severe roll velocities and angles than the Robin. The Mayfly concept was intended to outperform the Robin by having a lower center of gravity (hence preventing rollover), but the radically higher sprung : unsprung mass ratio means that the Mayfly is actually subjected to higher rolling velocity and angle than the Robin in the same cornering maneuver. Although the car doesn't leave the ground and rollover, this will be significantly more uncomfortable for the occupants and does not bode well for performance in other, more severe manoeuvres.

\section{Summary/Conclusion(s)}

The Mayfly concept incorporates hub motors, as it was thought that this would lower the centre of gravity and prevent rollover. It followed that the Mayfly should therefore perform better in cornering than existing delta-configuration three-wheeled cars. This analysis shows that this is not the case.

The use of hub motors doubles the sprung : unsprung mass ratio of the vehicle, and it consequently exhibits radically different behaviour to the Reliant Robin 850 used as a benchmark. The Mayfly body actually rolls in the opposite direction to that of the Robin during steady-state cornering, achieving nearly twice the roll angle and velocity (at 6 degrees and $30 \mathrm{mph}$ ).

The study is limited, since only steady state (and not transient) cornering is simulated, and the body is assumed to be rigid. However, incorporating a more demanding maneuver and a flexible body will likely yield even worse dynamic behaviour. The use of hub motors does provide scope for differential steering, which is not investigated here.

The Mayfly concept as it stands is unlikely to be marketable, certainly not to the aspirational racer market it is intended for. However, the analysis has been conducted sufficiently early in the design process to propose and implement significant changes. These changes could include:

- Switching from a 'delta' configuration (one wheel at the front) to a 'tadpole' configuration (two wheels at the front).

- Investigating different powertrain options so as to alter the sprung : unspung mass ratio.

- Changing the chassis dimensions, e.g. increasing the track width.

- Controlling the vehicle in cornering, using a tilting mechanism, differential steering, and/or torque vectoring.

\section{Recommendation}

The Mayfly concept with off-the-shelf hub motors and a Reliant Robin chassis cannot provide the desired driver experience. However, a model has been developed in this paper which can facilitate modelbased design of the Mayfly.

This study motivates a programme of further work aiming to develop the concept into a design with marketable performance. Using the model developed here, this will include a back-to-back comparison of a delta and tadpole configuration models, and an optimisation of the weight distribution and chassis dimensions. Once a satisfactory vehicle configuration is established, it can then be further enhanced by exploring the effects of tilting, differential steering and torque vectoring.

\section{References}

1. Berote, J., "Dynamics and Control of a Tilting Three Wheeled Vehicle (PhD Thesis)," University of Bath, Dept. Mechanical Engineering, Bath, UK., 2010.

2. Margetts, R., "Modelling the Dynamics of a Three-wheeled Racecar: A Pilot Study to Establish the Feasibility of Developing a 'Delta' Configuration Performance Car," Preprints, 2018.

3. Gillespie, T. D., Fundamentals of Vehicle Dynamics, Warrendale, PA.: Society of Automotive Engineers, Inc., 1992.

4. IPG Automotive, "CarMaker," IPG Automotive Gmbh, 2019. [Online]. Available: https://ipg-automotive.com/productsservices/simulation-software/carmaker/. [Accessed 14 August 2019].

5. Karnopp, D., Margolis, D., and Rosenberg,R., System Dynamics Modeling and Simulation of Mechatronic Systems, Hoboken, NJ.: John Wiley \& Sons, Inc., 2006.

6. QS Motor, "5000W 273 45H V2 Brushless DC Gearless Electric Car In Wheel Hub Motor," Alibaba Group, 2019. 
[Online]. Available:

https://www.aliexpress.com/item/32811491015.html.

[Accessed 14 August 2019].

7. Carfolio, “1981 Reliant Robin 850,” 2013. [Online]. Available: https://www.carfolio.com/specifications/models/car/?car=2261. [Accessed 25th Jan. 2018].

8. Doumiati, M., Charara, A., Victorino, A., Lechner, D., and Dubuisson, B., "Modeling of Tire and Vehicle Dynamics," in Vehicle Dynamics Estimation using Kalman Filtering, John Wiley \& Sons, Inc., 2012.

\section{Contact Information}

Rebecca Margetts, Ph.D., C.Eng, MIMechE

Phone: (+44) 1522-837951

E-mail: rmargetts@lincoln.ac.uk

\section{Acknowledgments}

The authors thank Mibrid (a trading name of GBA Technologies Ltd.) for providing the original research topic and access to data. This work was initially funded by a Mobility Award from the Lincoln Institute of Advanced Studies (LIAS).

\section{Nomenclature}

\begin{tabular}{|l|l|}
\hline$a$ & Distance from front axle to center of mass \\
\hline$\alpha$ & Slip angle \\
\hline$b$ & Distance from rear axle to center of mass \\
\hline$b_{s}$ & Damping coefficient \\
\hline$C$ & Slip stiffness (aka Cornering stiffness) \\
\hline$\delta$ & Steering angle of front wheel \\
\hline$f$ & [subscript] denotes front wheel \\
\hline$F$ & Force \\
\hline$g$ & Acceleration due to gravity \\
\hline$h_{g}$ & Height of the center of mass above the chassis \\
\hline$J$ & Moment of inertia \\
\hline
\end{tabular}

\begin{tabular}{|c|c|}
\hline$k$ & Spring stiffness coefficient \\
\hline$\mu$ & Friction coefficient \\
\hline$n$ & $\begin{array}{l}\text { [subscript] wheel position: can be front } f \text {, rear right } r r \\
\text { or rear left } r l \text {. }\end{array}$ \\
\hline$N$ & Normal Force \\
\hline$p$ & Generalized force \\
\hline$q$ & Generalized displacement \\
\hline$r l$ & [subscript] denotes rear left wheel \\
\hline$r r$ & [subscript] denotes rear right wheel \\
\hline$R w$ & Wheel radius \\
\hline$s$ & [subscript] denotes sprung mass \\
\hline$s$ & Gradient of tire slip curve \\
\hline$\theta$ & Angular displacement \\
\hline$U$ & Forward velocity \\
\hline us & [subscript] denotes unsprung mass \\
\hline$V$ & Lateral velocity \\
\hline$v_{g}$ & Vertical velocity of ground \\
\hline$W$ & Vertical Velocity \\
\hline$w$ & Track, i.e. distance between left and right rear wheels \\
\hline$\omega$ & Rotational velocity \\
\hline$p$ & [subscript] denotes pitch motion \\
\hline$r$ & [subscript] denotes roll motion \\
\hline$y$ & [subscript] denotes yaw motion \\
\hline$x$ & [subscript] denotes fore/aft (longitudinal) motion \\
\hline$y$ & [subscript] denotes lateral motion \\
\hline$z$ & [subscript] denotes vertical motion \\
\hline
\end{tabular}

\section{Definitions, Acronyms, Abbreviations}

BEV

Battery Electric Vehicle

ICE
Internal Combustion Engine 